\title{
Extraction of antibacterial active compounds from dry leaves of African plants of the Combretaceae family
}

\author{
Stefano Banfi', Luca Chiodaroli', Serena Cavallari', Enrico Caruso', Paola Barbieri', \\ Viviana Orlandi', Pierangelo Clerici' ${ }^{2}$, Paolo Viganò ${ }^{3}$ \\ I Università degli studi dell'Insubria, Dipartimento di Biologia Strutturale e Funzionale, Varese \\ 2 Ospedale civile di Legnano, U.O. di Microbiologia, Legnano \\ 3 Ospedale civile di Legnano, U.O. Malattie Infettive, Legnano
}

Key words: leaves, extraction, antibacterial activity, C. micranthum, G. senegalensis

Purificazione e attività antimicrobica di estratti ottenuti da foglie di piante africane appartenenti alla famiglia delle Combretaceae

\section{SUMMARY}

The dry leaves of two African plants of the Combretaceae family, furnished by the botanist of the St. Jean de Dieu hospital of Tangueità (Benin, central Africa), were extracted with a sequence of 5 solvents with increasing polarity (from cyclohexane to water). The raw materials, obtained from these extractions following solvent evaporations, were tested for antibiotic activity against gram negative and gram positive bacterial strains. According to the results of a modified Kirby-Bauer test, no promising effect was obtained against Gram negative bacteria while interesting dose-effect activities were observed against Gram positive strains. In particular, from G. senegalensis active compounds were found in the low polarity extract (dichloromethane) which, at a concentration of 800 $\mu \mathrm{g} /$ disk ( $13 \mathrm{~mm}$ diameter disk), resulted in a grow inhibition crown of $4.7 \mathrm{~mm}$ and $2.6 \mathrm{~mm}$ on Enterococcus faecalis and Staphylococcus aureus MSSA, respectively. An higher amount of the aqueous extract (4760 $\mu \mathrm{g} / \mathrm{disk})$ also produced a good result as $5.7 \mathrm{~mm}$ and $5.0 \mathrm{~mm}$ crowns were observed.

The extracts from C. micranthum showed an inhibiting effect in the more polar extracts (i.e. from ethanol and water) which gave I mm of grow inhibition crown on both strains at a concentration of $1000 \mu g / d i s k$.

The most promising extract from each plant was partially purified and then tested on some clinical relevant bacterial strains: S. aureus MRSA, Clostridium difficile, Streptococcus pyogenes, Streptococcus pneumoniae, Corynebacterium striatum, Neisseria gonorrhoeae, Haemophylus influenzae, Escherichia coli, obtaining a good killing effects on the Gram positive bacteria of the panel.

\section{INTRODUZIONE}

La medicina etnica Africana fa tutt'oggi ampio uso di decotti o infusi di foglie, radici e cortecce di varie piante locali la cui azione curativa verso diverse patologie è stata tramandata di generazione in generazione. Sorprendentemente queste tisane trovano impiego nei confronti di patologie tra loro estremamente diverse che vanno dai dolori addominali a sindromi respiratorie, dalle infezioni batteriche o protozoarie fino ad essere impiegate anche verso gravi patologie quali l'AIDS $(6,14)$. Quasi mai è conosciuto il principio attivo che origina l'effetto benefico osservato, tanto più che molte volte vengono fatti ingerire, o localmente applicati, miscugli di diverse piante o parti diverse della stessa pianta, quindi è facile presupporre che siano molteplici le sostanze assunte dagli ammalati sotto forma di tè, soluzioni idro-alcoliche, polveri o altro. Non è nemmeno certo che gli effetti curativi osservati non siano semplice causa di suggestione o, come si osserva, dovuti al semplice fatto che gli ammalati che riescono ad accedere alle strutture sanitarie trovano il conforto di cibo ed acqua in quantità ben maggiore di quanto siano abituati a ricevere nella loro vita rurale, con conseguente miglioramento generale del loro stato fisico.

$\grave{E}$ un fatto comunque noto che un gran numero dei farmaci oggi usati nei paesi sviluppati sono stati identificati in vegetali di origine africana o del centro/sud America e che tuttora la ricerca di nuove molecole "naturali" capaci di azione farmacologica specifica è particolarmente importante e perseguita (9). Per questa ragione, una valutazione accurata delle sostanze presenti nelle foglie, radici o cortecce di piante africane, opportunamente selezionate tra quelle usate nella medicina etnica locale, è importante per individuare nuovi farmaci. Questa necessità è particolarmente sentita nel campo degli antibiotici, genericamente intesi come sostanze antimicrobiche. Infatti, contrariamente a quanto si 
ipotizzò con l'avvento di un gran numero di nuovi antibiotici a partire dagli anni ' 60 , l'insorgenza di nuove e maggiori resistenze da parte di alcune famiglie batteriche, (quali ad esempio le Enterobacteriaceae e Pseudomonadaceae, di alcuni ceppi di S. pneumoniae, S. pyogenes e anche di Stafilococchi), rende spesso inefficace l'utilizzo degli antibiotici di vecchia generazione che sono $\mathrm{i}$ più diffusi e meno costosi (12). In questo lavoro si vogliono riportare i risultati preliminari nella ricerca di sostanze con attività antibatterica, studio di cui ci stiamo recentemente occupando $(2,3)$, effettuata su foglie secche di due piante tipiche della medicina etnica del Benin, stato dell'Africa sub sahariana, il Combretum micranthum e la Guiera senegalensis (entrambe appartenenti alla famiglia delle Combretaceae). L'estrazione dei principi attivi è stata eseguita mediante il trattamento delle foglie con una sequenza di solventi a polarità crescente in modo da estrarre, con ciascuno di essi, famiglie di sostanze con caratteristiche chimicofisiche simili discriminate in base alla compatibilità con la polarità del solvente.

\section{MATERIALI E METODI.}

Le foglie secche e intere di C. micranthum e $G$. senegalensis sono state fornite dalla sezione di botanica dell'ospedale St. Jean de Dieu di Tanguietà (Benin) in diversi periodi a cominciare dal 2008. I solventi usati (cicloesano, diclorometano, toluene ed etanolo) sono puri per analisi (Sigma-Aldrich) ed usati tal quali. Acqua distillata è stata usata per l'ultima fase di estrazione.

$\mathrm{La}$ fase stazionaria (silice, $\mathrm{SiO}_{2}$ ) usata per le cromatografie è un prodotto Fluka (silica gel 60; $0.063-0.2 \mathrm{~mm}$ ) le lastrine per la cromatografia su strato sottile (TLC) sono del tipo Fluka silica gel/TLC cards, spessore $0.2 \mathrm{~mm}$ (con indicatore di fluorescenza a $254 \mathrm{~nm}$ ). Le analisi HPLC sono state effettuate con uno strumento Agilent 1100 series con rivelatore Uv-vis a diodi (DAD), munito di colonna Supelcosil LC8 $(15 \mathrm{~cm})$ o Agilent C18 $(15 \mathrm{~cm})$, flusso di eluizione $0.5 \mathrm{ml} / \mathrm{min}$ (eluenti specificati nei singoli casi). Il terreno $\mathrm{MH}$-agar è stato usato per verificare la sensibilità dei ceppi batterici in esame ai singoli estratti secondo il metodo Kirby-Bauer (KB) come da protocollo CLSI opportunamente modificato.

Per uno screening iniziale dell'attività antibatterica delle frazioni grezze è stato usato un microrganismo Gram positivo (Staphylococcus aureus MSSA ATCC 25293) e uno Gram negativo Escherichia coli C1a. L'attività delle frazioni parzialmente purificate è stata ulteriormente valutata su una serie di ceppi batterici di interesse clinico: S. aureus MRSA, Clostridium difficile, Streptococcus pyogenes, Streptococcus pneumo- niae, Corynebacterium striatum, Neisseria gonorrhoeae, Haemophylus influenzae.

\section{Procedura di estrazione}

$100 \mathrm{~g}$ di foglie secche sono state sminuzzate a mano, versate in un pallone da 11 e trattate con il primo solvente, cicloesano (CHE, $600 \mathrm{ml}$ ), per 15 ore a temperatura ambiente sotto blanda agitazione meccanica (NB: una eccessiva polverizzazione delle foglie rende il materiale non agitabile). Dopo questo trattamento le foglie vengono filtrate e lasciate asciugare all'aria mentre il solvente viene recuperato a parte. Le foglie sono successivamente trattate con gli altri solventi [toluene (TO), acetone ( $\mathrm{AC})$, etanolo $(\mathrm{EtOH})$ e, per finire, con acqua $(\mathrm{W})$ ] nelle stesse quantità e con la stessa procedura. I solventi organici sono singolarmente evaporati in vuoto con l'ausilio di un evaporatore rotante mantenendo la temperatura del bagnomaria al di sotto dei $40^{\circ} \mathrm{C}$ in modo da minimizzare un eccessivo riscaldamento delle sostanze contenute negli estratti, così minimizzando una eventuale e indesiderata decomposizione dei principi attivi. L'estratto ottenuto con l'acqua è recuperato solido in seguito ad un processo di liofilizzazione che prevede il congelamento a $-80^{\circ} \mathrm{C}$ e sublimazione in vuoto con uno strumento VirtisSentry freeze dryer.

Dall'allontanamento dei solventi si ottengono dei residui grezzi che vengono pesati su bilancia analitica. I risultati per Guiera e Combretum sono riportati in Tabella 1.

\section{Purificazione parziale delle frazioni più attive di ciascuna pianta}

Nel caso di G. senegalensis l'estratto da TO (campione Gs2, Tabella 2) è risultato il più attivo nei confronti dei due ceppi batterici modello. Questa frazione è stata inizialmente purificata tramite procedure di estrazione acido-base (composto verde Gs2-AB, Schema1) e, in seguito, con una cromatografia su silice per fornire il composto finale (Gs2-P, Schema 2 e Tabella 3) che risulta quasi puro ad un'analisi su cromatografia su strato sottile (TLC, $\mathrm{SiO}_{2}-\mathrm{CH}_{2} \mathrm{Cl}_{2}$ con $0.4 \%$ acido acetico). Il prodotto viene analizzato con HPLC (colonna Agilent, eluente acetonitrile/metanolo $90 / 10+0.1 \%$ di acido acetico) dimostrando che è puro al $95 \%$. Con queste procedure da $1.5 \mathrm{~g}$ di grezzo di Gs2 si recuperano ca. $40 \mathrm{mg}$ di un composto verde. Per quanto riguarda $C$. micranthum, l'estratto con EtOH (Cm4-L, Tabella 1) è risultato il più efficace contro i due ceppi modello, quindi si è proceduto ad una sua purificazione mediante un lavaggio con etere etilico $(50 \mathrm{ml})$ seguito da solubilizzazione in fase acquosa a $\mathrm{pH}$ variabili da 4 a 7 . In questi passaggi si osserva che l'attività 
antibatterica è sempre riscontrata nella fase acquosa (Cm4-W, Schema 1). La fase acquosa viene liofilizzata ed il residuo solido giallino $(0.6 \mathrm{~g})$ viene trattato con acetone/metanolo: 8/2 (100 ml). L'attività risulta tutta e solo nella fase acetonica che una volta evaporata ha fornito ca. $300 \mathrm{mg}$ di un solido biancastro (punto di fusione $141^{\circ} \mathrm{C}$, molto igroscopico) che cristallizza in piccole sfoglie. Anche questa sostanza sembra sufficientemente pura ad un'analisi su TLC $\left(\mathrm{SiO}_{2}\right.$ - acetone/metanolo: 1/1) e ad un'analisi HPLC (colonna Supelcosil, eluente: acetonitrile) mostra una purezza del $96 \%$. Per la valutazione dell'attività antibatterica dei composti recuperati dai vari frazionamenti, si è proceduto sciogliendo ciascuna frazione in etanolo in modo da avere sempre soluzioni omogenee a concentrazione nota, usabili per il caricamento dei dischetti di carta per il test di sensibilità.

\section{Attività antibatterica}

L'effetto antibatterico delle singole frazioni è stato valutato utilizzando il metodo modificato di diffusione su agar (4): colture pure dei microrganismi scelti come tester sono state utilizzate per ottenere una sospensione pari a $0.5 \mathrm{McF}$ arland in soluzione salina e inoculate su terreno MuellerHinton agar. I dischetti di carta $\left(\mathrm{BBL}^{\mathrm{TM}} \mathrm{TAXO}^{\mathrm{TM}}\right.$ $\mathrm{BD})$ delle dimensioni di $13 \mathrm{~mm}$, imbevuti con un volume massimo totale di $500 \mu \mathrm{l}$ (ottenuto con sequenze di caricamento da $100-150 \mu \mathrm{l}$ ) delle varie soluzioni, sono stati attentamente asciugati con una corrente di aria calda fino a peso costante, quindi appoggiati sulle piastre di terreno Mueller-Hinton precedentemente seminate con il microrganismo di interesse e incubate a $37^{\circ} \mathrm{C}$ per $24 \mathrm{~h}$. L'effetto antibatterico è stato valutato misurando la dimensione della corona di inibizione della crescita batterica in quattro direzioni ortogonali tra loro in seguito a fotografia ad alto ingrandimento con l'uso di un software di elaborazione fotografica (GIMP) appositamente configurato. In questo modo si è potuto valutare in maniera estremamente precisa, nell'ordine dei 2 decimi di millimetro, ogni minimo effetto prodotto dagli estratti sui vari ceppi batterici. I risultati dell'attività dei grezzi, come valore medio di 4 misure ortogonali effettuate su tre prove per ogni estratto o campio- ne da valutare e la deviazione standard delle misure sono riportati in Tabella 2

I dati relativi all'azione antibatterica dei campioni purificati, valutata come riportato per i grezzi, sono riportati in Tabella 3.

\section{RISULTATI}

Dalle estrazioni successive con solventi organici ed acqua, a partire da $100 \mathrm{~g}$ di foglie secche di ciascuna delle due piante oggetto del presente studio, si sono ottenuti i seguenti grezzi (Tabella 1).

Per valutare l'attività antibatterica, di ciascun grezzo sono state preparate delle soluzioni a concentrazione nota e, per diminuire la variabilità eventualmente dovuta al tipo di solvente, i primi tre grezzi ottenuti da ciascuna pianta con $\mathrm{CHE}, \mathrm{TO}$ e AC, sono stati ripresi in diclorometano (DCM), mentre gli estratti da etanolo ed acqua sono stati nuovamente sciolti nei rispettivi solventi. Da queste soluzioni a concentrazione nota si sono potuti caricare i dischetti con le quantità opportune di ciascun estratto considerando che il volume massimo totale caricabile è risultato di $500 \mu \mathrm{l}$. Raggiunta la quantità desiderata di sostanza, i dischetti sono stati accuratamente asciugati e quindi appoggiati sulle piastre seminate. Per accertarsi che non vi fosse alcun effetto dovuto al solvente di caricamento, per ciascun solvente diverso dall'acqua, si sono preparati anche alcuni dischetti imbevuti con il solo solvente puro $(500 \mu \mathrm{l})$ e si è proceduto alla loro asciugatura. Nessun solvente puro lascia qualche residuo con azione antibatterica mentre l'effetto di alcuni estratti è stato chiaramente osservato su $S$. aureus (Figura I).

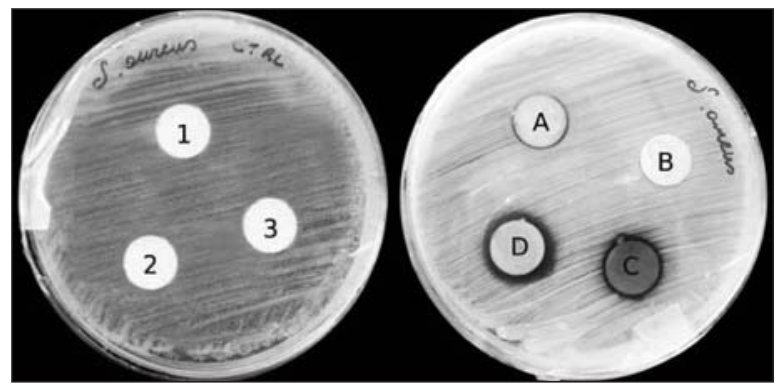

Figura I. A sinistra i controlli con DCM (I), $\mathrm{MeOH}(2)$ e acqua (3); a destra un esempio degli aloni ottenuti per Cm3 (A), Gs3 (B), Gs5 (C) e Gs2 (D) su S. aureus.

Tabella I. Peso del materiale grezzo ottenuto dalle estrazioni seriali con 5 solventi a partire da 100 g di foglie secche di Guiera senegalensis e Combretum micranthum

\begin{tabular}{|c|c|c|}
\hline Solvente & Estratto secco G. senegalensis & Estratto secco C. micranthum \\
\hline Cicloesano (CHE) & $2.2 \mathrm{~g}(\mathrm{Gsl})$ & $\mathrm{I} .5 \mathrm{~g}(\mathrm{Cm} \mathrm{I})$ \\
\hline Toluene (TO) & $1.5 \mathrm{~g}(\mathrm{Gs} 2)$ & $0.5 \mathrm{~g}(\mathrm{Cm} 2)$ \\
\hline Acetone (AC) & $2.5 \mathrm{~g}$ (Gs3) & $1.4 \mathrm{~g}(\mathrm{Cm} 3)$ \\
\hline Etanolo (EtOH) & $5.6 \mathrm{~g}(\mathrm{Gs} 4)$ & $6.7 \mathrm{~g}(\mathrm{Cm} 4)$ \\
\hline Acqua (W) & $6.6 \mathrm{~g}$ (Gs5) & $6.0 \mathrm{~g}(\mathrm{Cm} 5)$ \\
\hline Peso totale & $18.4 \mathrm{~g}$ & I6.I g \\
\hline
\end{tabular}


Purtroppo, usando quantità equivalenti dei vari estratti, nessun alone di inibizione di $E$. coli è visibile (Tabella 2).

Il maggior effetto di inibizione della crescita della colonia batterica di $S$. aureus è riscontrato con l'estratto grezzo Gs2 (da Guiera con Toluene) e con quello etanolico da Combretum (Cm4). Per meglio evidenziare gli effetti osservati si è proceduto ad una purificazione parziale di questi due grezzi con varie procedure chimiche che comprendono estrazioni e contro-estrazioni acqua/solvente, cromatografie e cristallizzazioni.

Nella Figura II si riportano i passaggi effettuati per la purificazione dei grezzi in TO di G. senegalensis e in EtOH di C. micranthum riportando le sigle delle frazioni di volta in volta ottenute.

La purezza delle sostanze recuperate dai processi di frazionamento è stata controllata via TLC e HPLC; per entrambe le frazioni finali (Gs2-P e $\mathrm{Cm} 4-\mathrm{P}$ ) la purezza è risultata superiore al $95 \%$.

Le frazioni purificate sono quindi state testate con il metodo di diffusione su agar su una serie di ceppi batterici di interesse clinico ed i risultati sono riportati in Tabella 3.

Come si può notare da entrambe le specie di Combretaceae si sono estratte sostanze con buona attività antibatterica su ceppi Gram positivi mentre praticamente nulla è l'azione su ceppi Gram negativi. Inoltre si osserva (Tabella 2) che nel caso del $C$. micranthum l'attività maggiore è riscontrata nella frazioni ottenute da solventi polari $(\mathrm{EtOH}>\mathrm{W})$ quindi riferibile ad una sostanza idrofila, mentre nel caso della G. senegalensis il dato più interessante è ottenuto con l'estratto lipofilo da toluene, così indicando che le molecole attive sono certamente di natura diversa.

\section{DISCUSSIONE}

In letteratura è possibile riscontrare un abbondante numero di lavori che analizzano l'attività di sostanze estratte da molte specie di Combretaceae, famiglia di piante che comprende almeno 300 specie del genere Combretum e 100 specie del genere Terminalia oltre a altre specie minori. Le molecole più comunemente riconosciute per la loro attività biologica sono appartenenti alla famiglia dei flavonoidi, polifenoli, tannini, stilbenoidi (combrestatine) e derivati dell'acido ellagico $(5,7,10)$.

Sebbene le Combretaceae siano tra le piante più studiate, in particolare le specie molle, erythrophyllum, imberbe e woodii, le specie C. micranthum e G. senegalensis trovano un limitato riscontro in letteratura dove sono riportate per attività antimalarica (13) e antivirale (8). A nostra conoscenza non vi sono riferimenti specifici ed evidenti di una possibile attività antibatterica ottenuta per estrazione delle foglie, sebbene il C. micranthum sia stato selezionato dall'associazione per gli standard delle piante medicinali africane (AAMPS) tra le 50 piante più importanti dal punto di vista della medicina etnica africana (7).

Noi abbiamo preso in considerazione queste due specie in quanto usate nella medicina tradizionale del Benin, come indicatoci dalla ONLUS Gruppo Solidarietà Africa (GSA) che opera presso l'ospedale di St. Jean de Dieu a Tanguietà e che ha fornito il materiale fogliare.

In letteratura sono riportate diverse procedure di estrazione di materiale fogliare che vanno dall'uso di miscele omogenee di acqua/alcool o solo alcool all'utilizzo di ulteriori miscele eterogenee di solvente organico/acqua (11). In questo lavoro

\section{Guiera senegalensis}

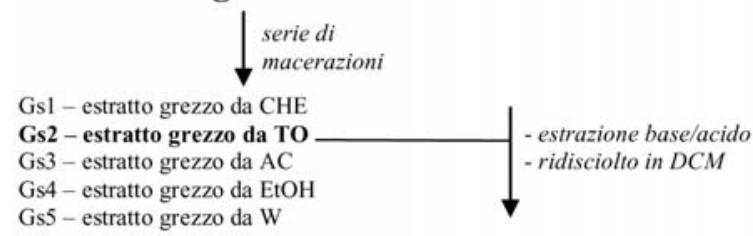
Gs5 - estratto grezzo da W

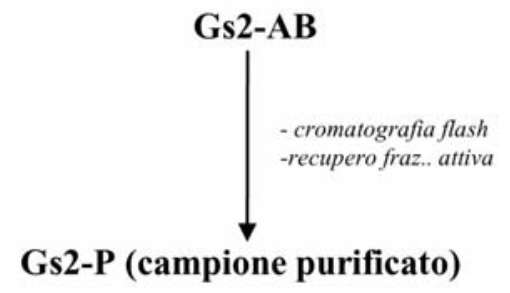

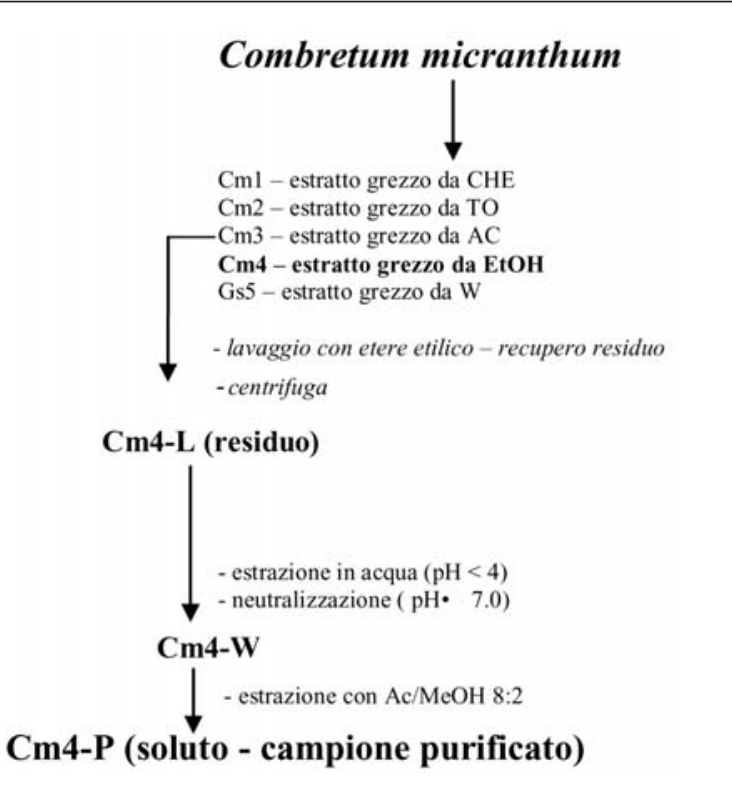

Figura II. 
abbiamo scelto di procedere all'estrazione delle foglie secche con la sequenza di solventi a polarità crescente, riportata in Tabella 1, similmente a quanto riportato da Eloff (1).

Come si vede dai dati riportati in Tabella 1, la quantità di sostanza estratta con i vari solventi dipende dalla polarità del solvente e che le maggiori quantità di grezzo sono state ottenute con $\mathrm{i}$ solventi più polari, infatti si passa dall' $1-2 \%$ del peso secco con CHE e TO per arrivare al $6-7 \%$ con EtOH e W. Il peso totale di sostanze recuperate dalle due specie è sostanzialmente equivalente e la differenza di ca. $2 \mathrm{~g}$ a favore di Guiera è sostanzialmente dovuta alla differenza di peso degli estratti in TO e AC di Combretum rispetto a quello di Guiera.

Tutti gli estratti sono stati recuperati come solidi e sciolti in un opportuno solvente per avere a disposizione soluzioni a concentrazione nota, così ottenendo cinque campioni grezzi da ogni pianta che sono stati testati per un primo screening su due microrganismi modello, $S$. aureus ed E. coli.

I risultati riportati in Tabella 2 indicano che non si è osservata attività contro $E$ coli, mentre si riconosce l'attività antimicrobica di tre estratti grezzi ottenuti da G. senegalensis e due da C. micranthum verso $S$. aureus. In particolare, nel caso di Guiera, risultano attivi gli estratti da TO, EtOH e W (Gs2, Gs4, Gs5) mentre, per quanto riguarda Combretum, hanno mostrato attività antibatterica gli estratti da EtOH e W $(\mathrm{Cm} 3, \mathrm{Cm} 4)$. La ripetizione delle procedure di estrazione su diversi campioni di foglie raccolte in diversi periodi tra il maggio 2008 e il Settembre 2010, portano a minime variazioni dei pesi dei singoli estratti grezzi e agli stessi risultati di attività.

Dalla Tabella 2 si evince che le frazioni più promettenti dal punto di vista dell'attività antibiotica sono rispettivamente Gs2 e $\mathrm{Cm} 4$ per Guiera e Combretum. Si è quindi deciso di procedere alla purificazione almeno parziale di questi grezzi nel tentativo di isolare uno o più principi attivi in essi contenuti. Nei processi di purificazione, ad ogni passaggio si è proceduto alla valutazione dell'effetto antimicrobico di ciascuna frazione con il metodo di diffusione in agar, in modo da recuperare sempre la frazione contenente la maggiore attività, fino ad ottenere $\mathrm{i}$ due campioni Gs2-P e Cm4-P.

Queste sostanze purificate e alcuni intermedi di purificazione dei composti di Combretum (Cm4$\mathrm{L}, \mathrm{Cm} 4-\mathrm{W})$, sono state testate sui due ceppi

Tabella 2. Corona circolare di inibizione su colture di S. aureus e E. coli, ottenuti con il metodo di diffusione in agar con dischetti di carta da $13 \mathrm{~mm}$ caricati con $500 \mu$ ldei vari estratti grezzi

\begin{tabular}{|c|c|c|}
\hline $\begin{array}{l}\text { Campione } \\
\text { (quantità) }\end{array}$ & $\begin{array}{l}\text { Attività su S. aureus } \\
\text { (raggio corona) }\end{array}$ & $\begin{array}{l}\text { Attività su E. coli } \\
\text { (raggio corona) }\end{array}$ \\
\hline Gs I (I.0 mg) & $0 \mathrm{~mm}$ & $0 \mathrm{~mm}$ \\
\hline Gs2 (0.8 mg) & $2.6 \mathrm{~mm}$ & $0 \mathrm{~mm}$ \\
\hline Gs3 (0.8 mg) & $0 \mathrm{~mm}$ & $0 \mathrm{~mm}$ \\
\hline Gs4 (I.0 mg) & $2.0 \mathrm{~mm}$ & $0 \mathrm{~mm}$ \\
\hline Gs5 (I.0 mg) & $1.5 \mathrm{~mm}$ & $0 \mathrm{~mm}$ \\
\hline $\mathrm{Cml}(1.0 \mathrm{mg})$ & $0 \mathrm{~mm}$ & $0 \mathrm{~mm}$ \\
\hline $\mathrm{Cm} 2(0.8 \mathrm{mg})$ & $0 \mathrm{~mm}$ & $0 \mathrm{~mm}$ \\
\hline $\mathrm{Cm}^{3}(0.8 \mathrm{mg})$ & $0 \mathrm{~mm}$ & $0 \mathrm{~mm}$ \\
\hline Cm4 (I.0 mg) & $2.5 \mathrm{~mm}$ & $0 \mathrm{~mm}$ \\
\hline Cm5 (I.0 mg) & $1.0 \mathrm{~mm}$ & $0 \mathrm{~mm}$ \\
\hline
\end{tabular}

Tabella 3. Corone circolari di inibizione e relative deviazioni standard campionarie (sx) osservate su colture di ceppi di interesse clinico, ottenute con il metodo di diffusione in agar con dischetti di carta da $13 \mathrm{~mm}$ caricati con le quantità riportate di estratti da Combretum (Cm4-L, Cm4-W, Cm4-P) e da Guiera (Gs2-P)

\begin{tabular}{|c|c|c|c|c|c|}
\hline $\begin{array}{l}\text { Ceppi batterici } \\
\text { utilizzati }\end{array}$ & $\begin{array}{l}\text { Cm4-L } \\
(3 \mathrm{mg})\end{array}$ & $\begin{array}{l}\text { Cm4-W } \\
\text { (Img) }\end{array}$ & $\begin{array}{l}\text { Cm4-P } \\
\text { (I mg) }\end{array}$ & $\begin{array}{l}\text { Gs2-P } \\
\text { (Img) }\end{array}$ & \\
\hline S. aureus MSSA & $6.4 \mathrm{~mm} \quad(\mathrm{sx}=0.5 \mathrm{I})$ & $5.5 \mathrm{~mm} \quad(\mathrm{sx}=0.53)$ & $7.5 \mathrm{~mm}(\mathrm{sx}=0.50)$ & $9 \mathrm{~mm}$ & $(s x=0.74)$ \\
\hline S. aureus MRSA & $(s x=0.45)$ & $6.5 \mathrm{~mm} \quad(\mathrm{sx}=0.50)$ & $7.5 \mathrm{~mm}(\mathrm{sx}=0.56)$ & $9 \mathrm{~mm}$ & $(s x=0.8 I)$ \\
\hline C. difficile & $(s x=0.32)$ & $6.5 \mathrm{~mm} \quad(\mathrm{sx}=0.35)$ & $(s x=0.30)$ & $\mathrm{I} \mathrm{mm}$ & $(s x=0.20)$ \\
\hline S. pyogenes & $0 \mathrm{~mm}$ & $0 \mathrm{~mm}$ & $0 \mathrm{~mm}$ & $2.5 \mathrm{~mm}$ & $(s x=0.26)$ \\
\hline S. pneumoniae & $0 \mathrm{~mm}$ & $0 \mathrm{~mm}$ & $0 \mathrm{~mm}$ & $1.5 \mathrm{~mm}$ & $(s x=0.3 I)$ \\
\hline C. striatum & $1.4 \mathrm{~mm} \quad(\mathrm{sx}=0.26)$ & $(s x=0.38)$ & $(s x=0.25)$ & $2 \mathrm{~mm}$ & $(s x=0.44)$ \\
\hline N. gonorrhoeae & $0 \mathrm{~mm}$ & $0 \mathrm{~mm}$ & $0 \mathrm{~mm}$ & $0 \mathrm{~mm}$ & \\
\hline H. influenzae & $0 \mathrm{~mm}$ & $0 \mathrm{~mm}$ & $0 \mathrm{~mm}$ & $0 \mathrm{~mm}$ & \\
\hline E. coli & $0 \mathrm{~mm}$ & $(s x=0.37)$ & $(s x=0.3 I)$ & $0 \mathrm{~mm}$ & \\
\hline
\end{tabular}


modello e su una serie di microrganismi ritenuti importanti dal punto di vista clinico. I risultati riportati in Tabella 3 indicano che l'attività dei grezzi riscontrata su $S$. aureus viene confermata nei composti purificati con aloni circa 3 volte superiori a parità di quantità caricata (Tabella 2, riga 2 e 9 rispetto Tabella 3 , riga 1, colonna 4 e 5), inoltre per Combretum si osserva che anche i composti intermedi danno un'attività interessante (Tabella 3, riga 1, colonne 2 e 3). Particolarmente interessante appare il riscontro di attività su $E$. coli da parte del Cm4-P (Tabella 3, riga 9, colonna 4 ), attività che non si era potuto riscontrare con il grezzo corrispondente.

Per quanto riguarda gli altri ceppi batterici, con Gs2-P si ottiene una buona risposta antibatterica su tutti i Gram positivi (Tabella 3, righe 2-6, colonna 5) mentre rimane nullo l'effetto sui Gram negativi. Invece, il composto purificato da Combretum (Cm4-P) mostra attività su quattro dei sei Gram positivi (Tabella 3, righe 1-3,6) e su $E$. coli (come evidenziato sopra) ma non sugli altri due ceppi Gram negativi.

Particolarmente interessante è l'azione di entrambi composti purificati su $C$. difficile, che provoca infezioni nosocomiali importanti.

Il fatto che i composti da noi isolati risultino attivi su $S$. aureus sia MSSA che MRSA indica chiaramente che il meccanismo di azione è diverso da quello della Meticillina quindi questi composti potrebbero risultare particolarmente interessanti da un punto di vista clinico.

In conclusione si può affermare che sia C. micranthum che G. senegalensis contengono composti farmacologicamente interessanti per la loro azione antibatterica. Studi successivi saranno necessari per purificare completamente le sostanze in modo da determinarne la struttura chimica, inoltre sarà opportuno mettere a punto un procedimento che permetta di valutare i valori di MIC (minima concentrazione inibente) da mettere in relazione con gli aloni osservati con il metodo di diffusione su agar.

\section{RINGRAZIAMENTI}

Si ringrazia la ONLUS "Gruppo Solidarietà
Africa" (GSA - Seregno, Milano) per il supporto finanziario e la fornitura del materiale vegetale che hanno permesso lo svolgimento di questa ricerca.

\section{BIBLIOGRAFIA}

1. Angeh JE, Huang X, Sattler I, et al. Antimicrobial and anti-inflammatory activity of four known and one new triterpenoid from $C$. imberbe (Combretaceae). J Ethnopharm 2007; 110: 56-60.

2. Chiodaroli L, Banfi S, Caruso E, et al. Purificazione e attività antimicrobica di estratti derivanti da piante africane. XXXIX Congresso Nazionale AMCLI, Rimini, 20-22 ottobre, 2010.

3. Chiodaroli L, Cavallari S, Caruso E, Banfi S, Barbieri P, Orlandi V. Antimicrobial activity of African traditional plants. $28^{\text {th }}$ National Meeting Società Italiana di microbiologia generale e biotecnologie microbiche, Spoleto (TR) 11-13 giugno, 2009.

4. Clinical and Laboratory Standards Institute (CLSI). Performance Standards for Antimicrobial disk susceptibility tests. Approved Standard M2-A7. $11^{\text {th }}$ ed. CLSI Wayne, Pa: 2005.

5. Cragg GM, Newman DJ. Plants as a source of anticancer agents. J Ethnopharm 2005; 100: 72-9.

6. Eloff JN, Famakin JO, Katerere DPR. Isolation o fan antibacterial stilbene from $C$. woodii (Combretaceae) leaves. African J Biotech 2005; 4 (10): 1167-71.

7. Eloff JN, Katerere DR, McGaw LJ. The biological activity and chemistry of the southern African Combretaceae. J Ethnopharm 2008; 119: 686-99.

8. Ferrea G, Canessa A, Sampietro F, Cruciani M, Romussi G, Bassetti D. In vitro activity of a Combretum micranthum extract against herpes simplex virus types 1 and 2. Antiv Research 1993; 21: 317-25.

9. Koehn FE, Carter GT. The evolving role of natural products in drug discovery. Nature Rev. Drug Discovery 2005; 4: 206-20.

10. Kuete V, Tabopda TK, Ngameni B, Nana F, Tshikalange TE, Ngadjui BT. Antimycobacterial, antibacterial and antifungal activities of Terminalia superba (Combretaceae). South African J. Botany 2010; 76: 125-31.

11. Martini N, Eloff JN. The preliminary isolation of several antibacterial compounds from $C$. erythrophyllum (Combretaceae). J Ethnopharm 1998; 62: 255-63.

12. Neu HC. The crisis in antibiotic resistance. Science 1992; 257 (5073): 1064-73.

13. Soh PN, Benoit-Vical FJ. Are west african plants a souce of future antimalarial drugs? J Ethnopharm 2007; 114: 130-40.

14. Van Vuuren SF, Naidoo D. An antimicrobial investigation of plants used traditionally in southern Africa to treat sexually transmitted infections. J Ethnopharm 2010; 130: 552-8. 